\title{
Genetic Regulation of Puberty Timing in Humans
}

\author{
Felix R. Day John R.B. Perry Ken K. Ong \\ MRC Epidemiology Unit, Institute of Metabolic Science, University of Cambridge, Cambridge Biomedical \\ Campus, Cambridge, UK
}

\section{Key Words}

Puberty - Genetics · Menarche - Imprinted genes .

Body mass index - Obesity

\begin{abstract}
Understanding the regulation of puberty timing has relevance to developmental and human biology and to the pathogenesis of various diseases. Recent large-scale genome-wide association studies on puberty timing and adult height, body mass index (BMI) and central body shape provide evidence for shared biological mechanisms that regulate these traits. There is a substantial genetic overlap between age at menarche in women and BMI, with almost invariable directional consistency with the epidemiological associations between earlier menarche and higher BMI. By contrast, the genetic loci identified for age at menarche are largely distinct from those identified for central body shape, while alleles that confer earlier menarche can be associated with taller or shorter adult height. The findings of population-based studies on age at menarche show increasing relevance for other studies of rare monogenic disorders and enrich our understanding of the mechanisms that regulate the timing of puberty and reproductive function.
\end{abstract}

(c) 2015 S. Karger AG, Basel

\section{Introduction}

Puberty is the process of attainment of reproductive maturity and involves a range of physiological changes. The timings of specific pubertal milestones serve as markers of the tempo of development. Age at menarche, a woman's first menstrual bleed, is reasonably well recalled and is widely recorded in epidemiological studies. It has an estimated heritability of $\sim 50 \%$ in contemporary western settings [1] and is associated with later life risks for type 2 diabetes, cardiovascular disease and various other health outcomes $[2,3]$. The lack of similar wellrecalled pubertal measures in men means that similar data are still sparse. Earlier puberty timing is generally, but not invariably, associated with higher risks for adverse health outcomes, and therefore the global declines in average ages of puberty in females [4-9] and males [10] have important relevance for health. These secular trends appear to be driven by increases in childhood body mass index (BMI), which to some extent may underlie many of the later health risks $[10,11]$. Recent genetic studies have shed light on the mechanisms linking weight status to puberty timing, as well as other biological pathways that might independently link puberty timing to later health.

\section{KARGER 125}

E-Mail karger@karger.com www.karger.com/nen (c) 2015 S. Karger AG, Basel

0028-3835/15/1024-0247\$39.50/0
Ken K. Ong

MRC Epidemiology Unit, Institute of Metabolic Science

University of Cambridge, Cambridge Biomedical Campus Box 285

Cambridge CB2 0QQ (UK)

ken.ong@mrc-epid.cam.ac.uk 


\section{Rare Genetic Disorders of Puberty}

Rare mutations in $>20$ genes that disrupt the gonadotropin-releasing hormone ( $\mathrm{GnRH})$ axis have been described [12]. Those that disrupt embryonic migration of GnRH-producing cells from the nasal placode also cause anosmia (Kallmann syndrome) [13]. Mutations in other genes that encode hypothalamic neurotransmitters or their receptors, rather than GnRH cell migration, typically cause normosmic hypogonadotropic hypogonadism. These include the genes for kisspeptin (KISS1), neurokinin B (TAC3) and GnRH (GNRH1) and their receptors (KISSR, TACR3 and GNRHR). Hypogonadotropic hypogonadism associated with other pituitary hormone deficiencies also results from mutations that disrupt pituitary development, such as in HESX1 and SOX2.

Rare mutations in the genes that encode the pituitary hormones follicle-stimulating hormone (FSH) and luteinizing hormone (LH) have differing effects in males and females. In females, absent puberty and ovarian failure are consequences of deleterious mutations in the FSH $\beta$-subunit [14] or FSH receptor genes (FSHR) [15]. By contrast, affected males have reduced spermogenesis without other features of gonadal failure [16]. Only 1 consanguineous kindred is reported with a deleterious mutation in the $L H$ gene [17]. The homozygous proband had bioinactive $\mathrm{LH}$ and absent puberty at age 17 , while heterozygous males reported variable infertility but normal puberty, and heterozygous females had normal sexual development and were fertile.

Few genetic disorders are described that result in precocious puberty. As a mirror image of the hypogonadotropic hypogonadism phenotype due to loss of function mutations in KISS or KISSR, activating mutations in these genes result in precocious puberty, at least in girls [18, 19]. A recent study of 40 members of 15 families affected by precocious puberty used whole-exome sequencing to identify paternally inherited mutations in MKRN3 [20], an exclusively paternally expressed gene located in the imprinted Prader-Willi syndrome region. Since that report of deleterious mutations in 15 affected members of 5 families from Brazil, USA and Europe [20], MKRN3 mutations have also been described in $2 / 6$ affected families [21] and in 8/215 apparently sporadic cases [22]. Studies in mice indicate that MKRN3 may act as a hypothalamic repressor of puberty onset [20].

Recently, various genetic disorders of puberty associated with ataxia have been described. Rare mutations in POLR3A that disrupt RNA polymerase cause hypomyelinating leukodystrophy [23]. The combination of ataxia and hypogonadism is also seen in disorders of ubiquitination due to rare mutations in RNF216 and OTUD4 [24] and in individuals with Gordon Holmes syndrome as a result of mutations in CHIP (also called STUB1) [25, 26].

\section{Genetic Disorders of Both Puberty and Adiposity}

The adipocyte-derived hormone leptin and its receptor link nutritional status to the GnRH axis. Rare deleterious mutations in the leptin gene (LEP) cause severe earlyonset obesity with hyperphagia [27]. Furthermore, as in the mouse model [28], affected individuals have hypogonadotropic hypogonadism [29,30]. Leptin therapy in children with leptin deficiency allows normal puberty timing rather than precocious puberty [31], suggesting that leptin is necessary for $\mathrm{GnRH}$ axis activity but is not itself the trigger for puberty timing [32]. Rare deleterious mutations in LEPR cause severe, early-onset obesity and an apparently less severe reproductive phenotype of abnormally delayed rather than absent puberty, with irregular menses from age 20 years and normal adult estradiol, LH and FSH levels reported in 3 affected women [33]. Converse to those disorders of delayed puberty and excess adiposity, rare mutations in POLD1 were recently reported to cause hypogonadism in males associated with lipodystrophy (particularly lack of subcutaneous adipose tissue) and deafness [34]. Affected individuals have low BMI and limb muscle mass but are insulin resistant. POLD1 regulates DNA polymerase activity, but it is yet unclear how this relates to disruptions in gonadal function and adipose tissue development and metabolism.

\section{Recent Genome-Wide Association Studies}

In contrast to the long history of reports of mutations in cases and families affected by rare clinical disorders, the discovery of common genetic variants that contribute to the wide normal variation in puberty timing, as well as other complex physiological traits, has accelerated only in the last 5-10 years since the advent of genome-wide association studies (GWAS). This approach allows the concurrent analysis of millions of single nucleotide polymorphisms (SNPs) across the genome in very large individual-based study designs to provide the statistical power that is necessary to robustly identify genomic regions of association.

Four recent large-scale GWAS have been reported on puberty timing, adiposity and adult height (table 1). A GWAS for age at menarche identified 123 independent 
Fig. 1. Schematic diagram of the biology of puberty initiation. Knowledge from rare and monogenic studies is bound in red; the suggested mechanisms of common variants are bound in green.

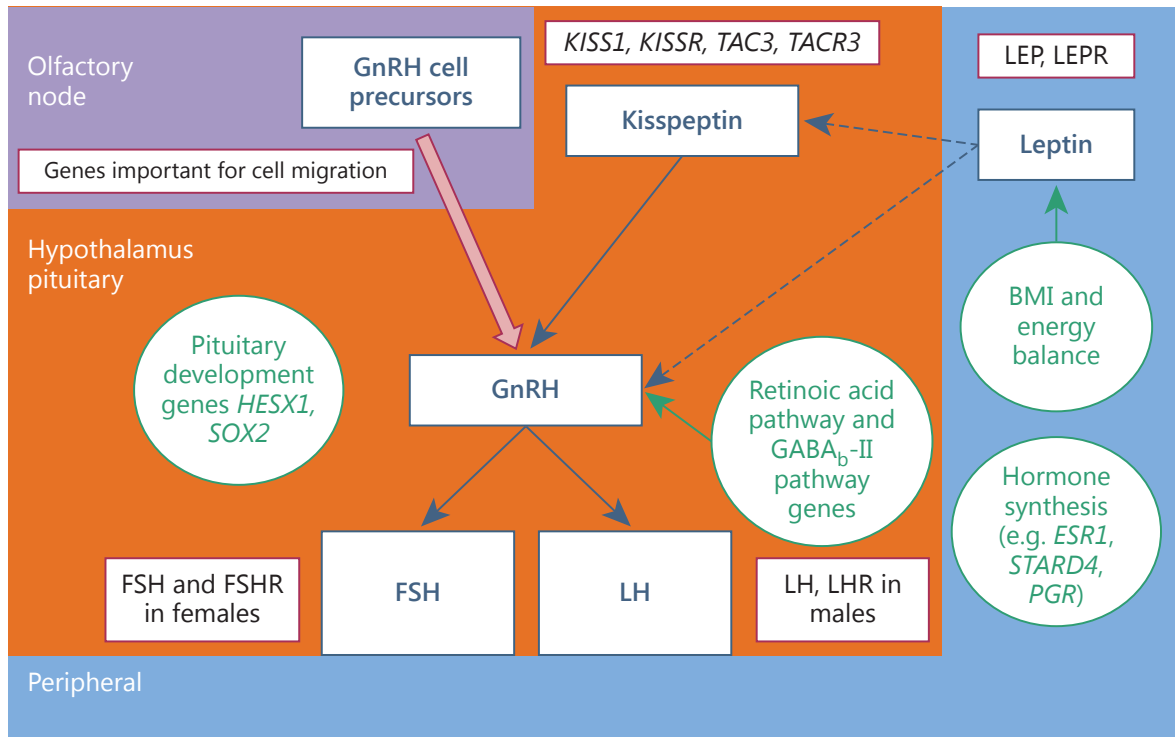

Table 1. Recent GWAS for puberty timing and anthropometric outcomes

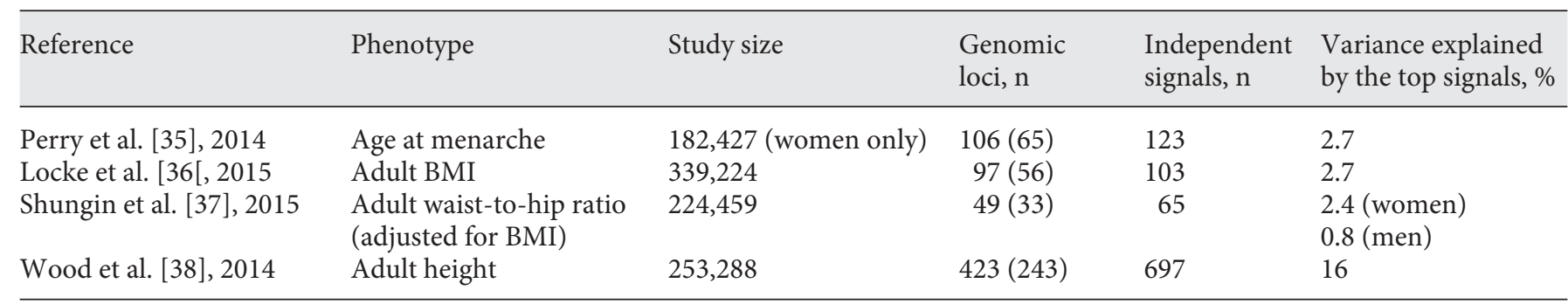

Genomic loci: values in parentheses indicate the number of novel loci.

signals at 106 genomic loci [35]. The strongest individual signal, an SNP near to $L I N 28 B$, altered age at menarche by 0.11 years per allele, and in total the top 123 SNPs explained $2.7 \%$ of the population variance in puberty timing. Secondly, a GWAS for BMI in adults identified 97 genomic loci, of which 56 had not been previously linked to any measure of adiposity. Taken together, the top 97 SNPs explained around $2.7 \%$ of the variance in adult BMI [36]. Thirdly, a parallel project by the same investigators identified 49 genomic loci, of which 33 were novel, robustly associated with measures of central-to-peripheral distribution of body shape and adiposity. The effects were greater in women than in men, probably reflecting the greater heritability in hip circumference in women. Together, the top SNPs explained $2.4 \%$ of the variance in 'waist-to-hip ratio adjusted for BMI' in women compared to only $0.8 \%$ of the variance in this trait in men [37]. Fi- nally, a remarkable 697 independent signals at 423 loci were associated with adult height. Together, these top SNPs explained $16 \%$ of the variance in adult height, and the investigators extrapolated that thousands of common variants across the genome account for $\sim 50 \%$ of variance [38].

Notably, all of these recent GWAS relied on traits that were measured or self-reported by adults, reflecting the relative paucity of genetic data in children, and therefore the role of these genetic variants in detailed childhood phenotypes is mostly unclear. A recent GWAS for childhood timing of 'Tanner puberty stages' in 6,147 girls and 3,997 boys did not identify any new loci for puberty timing but confirmed the relevance of the LIN28B locus [39], and the same data set supported the likely relevance of the vast majority of those loci recently identified for age at menarche in women to puberty timing in general in both boys 


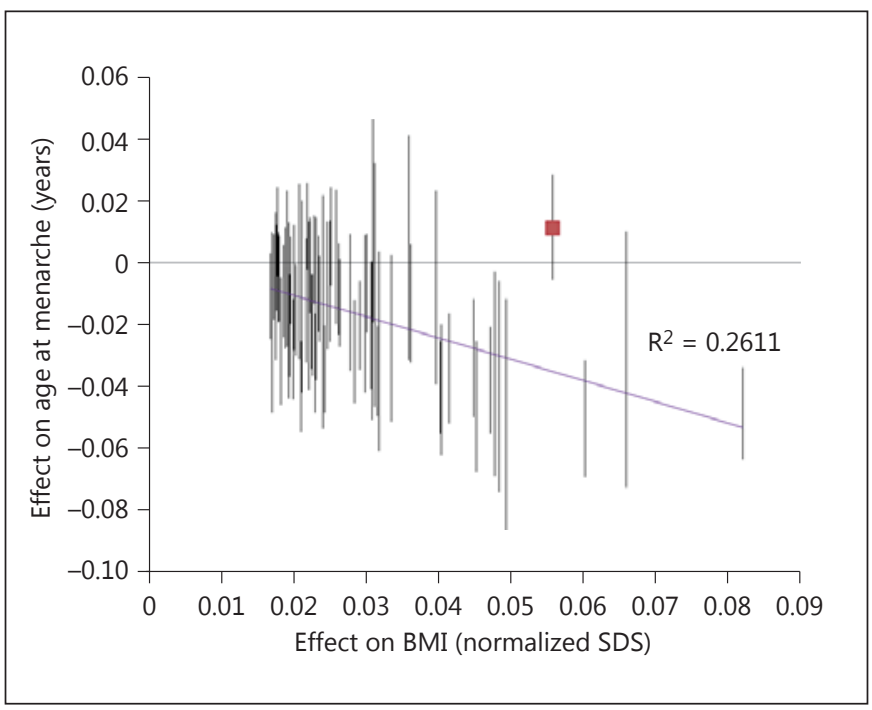

Fig. 2. Plot of the 97 common variants identified by GWAS for BMI, indicating their per-allele effect sizes on adult BMI (with 95\% confidence intervals) compared to their effect sizes on age at menarche. SDS = Standard deviation scores. The $\mathrm{R}^{2}$ value indicates the strength of correlation between effect sizes. In general, BMI-increasing alleles also confer earlier menarche. The $M C 4 R$ region variant (highlighted in red) is a notable exception.

and girls [35]. The reported menarche GWAS loci were enriched in/near genes that underlie rare disorders of puberty, including common variants in/near LEPR, GNRH1, PCSK1, PCSK2, POU1F1, TENM2, and TACR3, as described above, which indicates their likely generic relevance to puberty timing and reproductive function (fig. 1).

\section{Genetic Co-Regulation of BMI and Puberty Timing}

The existence of genetic co-regulation of BMI and age at menarche has been suggested by twin studies [40], and this prediction has been validated in recent GWAS findings. The list of shared loci between these two traits includes 13 genomic regions in or near the following genes: FTO, SEC16B, TMEM18, NEGR1, TNNI3K, GNPDA2, BDNF, BCDIN3D, GPRC5B, GALNT10, MAP2K5, TRIM66 and LRP1B. At all of these loci, the BMI-increasing allele is also associated with earlier age at menarche, which is directionally concordant with the observed epidemiologic association between BMI and age at menarche. In addition to these 13 'top signals' identified as passing stringent statistical criteria for each trait, our analysis across all of the 97 recently reported BMI loci shows an overall linear correlation between the reported effects on BMI [36] and those reported on age at menarche [35] $\left(R^{2}=0.26\right)$, indicative of a general mechanism linking these two traits (fig. 2).

However, there are some exceptions to this pattern, which could indicate other specific mechanisms that act on BMI and puberty timing. Firstly, the BMI locus near $M C 4 R$ has the fourth largest estimated effect size on BMI [41] (and has similar effects on BMI in men and women [42]) but shows no association at all with age at menarche $(\mathrm{p}=0.19$ [35]) (fig. 2), which suggest that it regulates BMI through a mechanism different to most other genetic factors. Intriguingly, at this locus the BMI-increasing SNP is also associated with later puberty in boys $(\mathrm{p}=0.0009)$ [39]. Further genetic studies in boys might shed light on the highly variable reported effects of BMI on puberty timing in observational epidemiological studies $[43,44]$. Secondly, a locus for age at menarche is located close to the BMI locus at ETV5. However, the genetic signals for these traits are not correlated, which may indicate heterogeneity in downstream biological mechanisms [45]. Lastly, our analysis of these reported findings and other publicly available data sets indicates that loci that regulate both BMI and age at menarche are also enriched for associations with fasting insulin levels, which could indicate a specific mechanism that potentially links these two traits (fig. 3).

In contrast to the reported BMI loci, the genetic loci reported for measures of central-to-peripheral distribution of body shape and adiposity show little relevance for age at menarche [37]. Only 1 locus for waist-to-hip ratio adjusted for BMI (at LEKR1) is also identified as a locus for age at menarche [35]. Notably, this LEKR1 locus is also reported to regulate birth weight [46], and the menarche age-raising allele has also been associated with higher birth weight, which is directionally concordant with observed epidemiological observation [47]. Therefore, while earlier age at menarche might predispose to greater central adiposity through its effects on promoting weight gain and BMI [48], GWAS findings do not yet support any distinct mechanisms linking these traits.

\section{Genetic Co-Regulation of Height and Puberty Timing}

There is good evidence from observational studies in the general population that, as is seen in girls with the clinical disorder of precocious puberty [49], girls who develop puberty earlier than average are taller than other girls during childhood and adolescence but are shorter as adults due to earlier epiphyseal bone fusion [50]; in con- 
Fig. 3. Heat map indicating the effects of 123 common variants identified for age at menarche on a number of metabolic-related phenotypes (data derived from publicly available data sets $[41,46$, 74-78]). GWAS Sig. $=\mathrm{p}<5 \times 10^{-8} ;$ Bon. Sig. $=p<5 \times 10^{-8}$; Nom. Sig. $=p<0.05$. Green bars indicate menarche age-decreasing variants that are also associated with higher values of other phenotypes; blue bars indicate menarche agedecreasing variants that are also associated with lower values of other phenotypes.

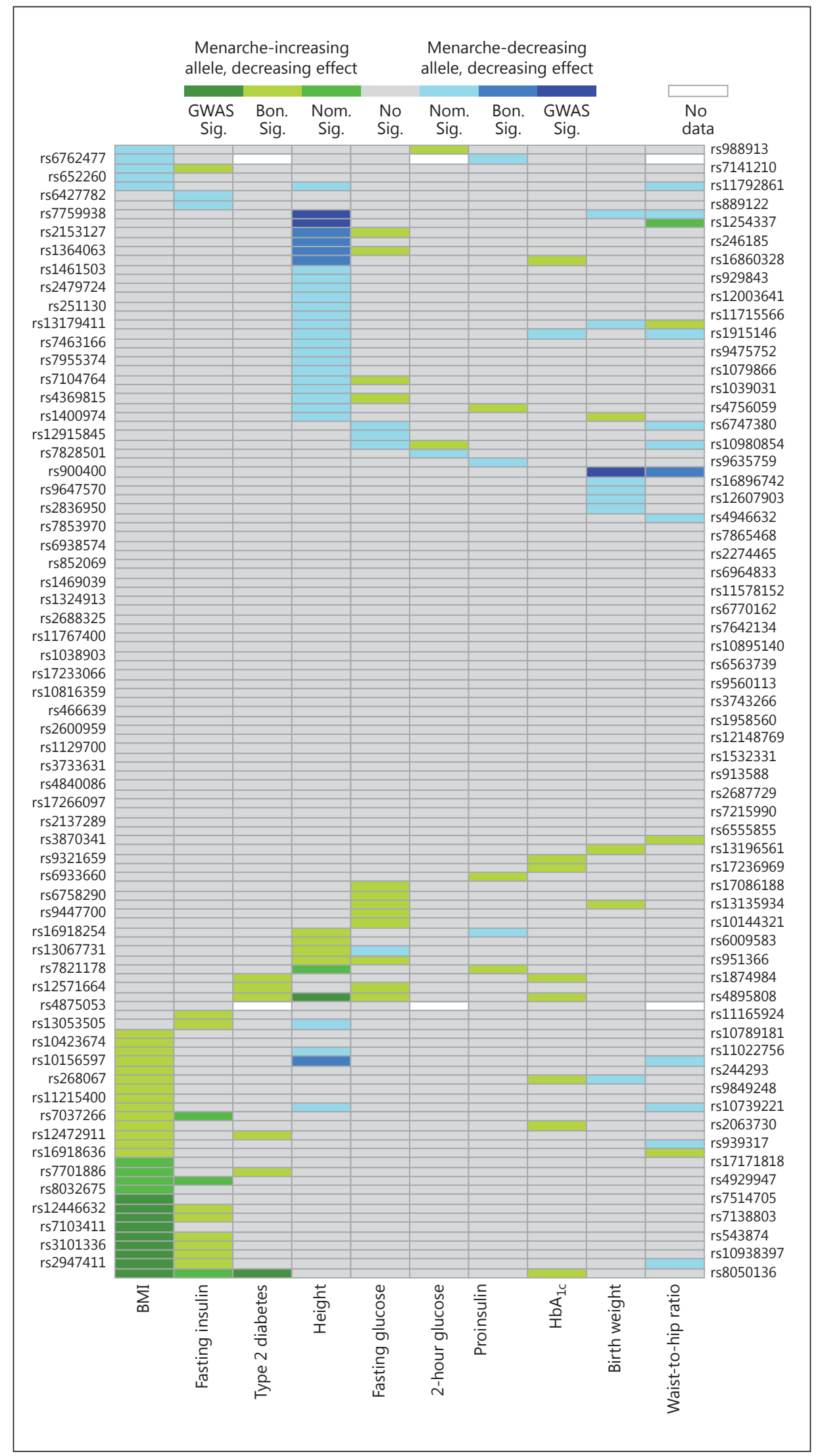


trast, there appears no association with adult height in men [51]. This complex relationship between height and puberty timing in females is reflected in the genetic coregulation of these traits. The GWAS findings for age at menarche include 3 loci that were also reported as GWAS loci for adult height; however, there was directional inconsistency in these overlapping findings. The menarche age-raising alleles at the LIN28B and SIX6 loci are also associated with taller adult height, which is directionally concordant with the phenotypic association. Conversely, at the $C E N P W / N C O A 7$ locus, the menarche age-raising allele is also associated with shorter adult height. Our analysis across all 123 signals identified for age at menarche shows distinct clusters of age at menarche alleles that are associated with either taller or shorter adult height (fig. 3). Clarification between such mechanisms, which promote both faster childhood growth and earlier puberty timing but have distinct effects on adult height potential, could have major clinical and public health benefits.

\section{Epigenetic Regulation of Puberty Timing}

The recent GWAS for age at menarche in women identified loci at 3 imprinted gene regions, MKRN3-MAGEL2, $D L K 1$ and KCNK9, which showed parent-of-origin-specific associations that were concordant with the established parent-of-origin-specific mRNA expression patterns. Thus, at the exclusively paternally expressed loci (MKRN3-MAGEL2 and DLK1) only the paternally inherited alleles (but not the maternally inherited alleles) showed associations with menarche timing, and vice versa for the exclusively maternally expressed locus KCNK9. The association pattern at the MKRN3-MAGEL2 locus is also concordant with the paternal-specific inheritance of rare pathological mutations in MKRN3 [20]. Notably, a recent parent-of-origin-specific GWAS for BMI identified 2 signals, including 1 near to KCNK9 [52]. Intriguingly, both paternally and maternally inherited alleles at KCNK9 are associated with BMI, but in opposite directions, and this SNP (rs2471083) is completely unrelated to the SNP identified for age at menarche (rs1469039; linkage disequilibrium $\mathrm{r}^{2}=0.001$ ). Therefore, there may be distinct biological mechanisms regulated by this same imprinted region.

More widely, there was enrichment of the 106 menarche-associated regions in known imprinted gene regions [35]. The importance of imprinting on pathways relating to energy balance in animal models [53] suggests that there may be further important motifs that also affect reproduction in humans. These findings indicate that the expression and phenotypic manifestation of some genetic factors that influence puberty timing are tightly regulated by stable epigenetic marks that reflect parental origin.

In addition to gene imprinting, the recent GWAS findings strongly indicate the potential involvement of other epigenetic mechanisms in puberty timing. These include JmjC-domain-containing demethylases that catalyze gene-activating epigenetic changes specifically at lysine 9 histone H3 marks [35]. Furthermore, a menarche-associated locus was identified at $C B X 7$, which encodes a component of the Polycomb group of transcriptional silencers [54]. These GWAS findings support the likely relevance in humans of the reported close epigenetic regulation of reproductive timing in animal models. $\mathrm{Pu}$ bertal onset in female rats is reportedly triggered by DNA methylation and decreased hypothalamic expression of 2 key Polycomb group genes, Eed and $C b x 7$, which in turn leads to the enrichment of activating lysine modifications on histone $\mathrm{H} 3$ and activation of the expression of Kiss 1 [54]. Studies in rhesus monkeys suggest that methylation and histone modification of the $G n R H$ gene itself affect expression [55]. Beyond the completion of puberty and the attainment of sexual maturity, epigenetic mechanisms may also contribute to the regulation of reproductive function, with epigenetic fluctuations observed across the female ovulation cycle [56].

\section{Other Mechanisms Inferred by GWAS}

In addition to those menarche GWAS loci identified in/near genes that underlie rare disorders of puberty, other menarche loci point to potential new candidate genes or biological pathways for such disorders. A menarche locus on chromosome 5 lies near PCSK1, in which rare mutations cause severe early-onset obesity and delayed/ absent puberty, as described in the 1 reported adult case [57]. The further menarche locus on chromosome 20 near PCSK2 strongly points to a common role of the encoded type 1 and 2 prohormone convertases in the regulation of puberty timing and reproductive function. Other menarche loci indicate candidate genes for $\mathrm{GnRH}$ neuron migration and pituitary development not yet linked to Kallmann syndrome and other hypothalamic/pituitary disorders. rs9647570 on chromosome 5 lies within TENM2, which encodes a transmembrane protein expressed in the developing brain and is induced by the product of the Kallmann syndrome gene FGF8 [58]. 
rs2479724 on chromosome 6 lies near FRS3, which encodes fibroblast growth factor receptor substrate 3 and is involved in the signalling of the product of the Kallmann syndrome gene FGFR1 [59]. rs7103411 and rs1129700 are menarche signals strongly associated with expression levels of LGR4 and TBX6, respectively, which are both genes that encode enhancers for the pituitary development factor SOX2. We anticipate that further overlap with GWAS findings will be uncovered, particularly as studies of rare disorders incorporate exome- and genome-wide sequencing approaches.

GWAS menarche loci were highly enriched in/near genes that encode nuclear hormone receptors, co-activators or co-repressors, including those receptors involved in retinoic acid signalling. The cluster of retinoid receptors comprises the retinoic acid receptors (RARA, RARB and RARG), the retinoic X receptors (RXRA, RXRB and RXRG) and also the retinoic acid receptor-related orphan receptors (RORA, RORB and RORC). They bind to retinoic acid response elements, activate gene transcription and have diverse functions across embryonic development (including GnRH neuron migration [60]), cell differentiation and homeostasis (including $\mathrm{GnRH}$ expression and secretion [61]).

In addition to the substantial role of genetic determinants of obesity susceptibility on puberty timing, our findings indicate other mechanisms that could explain the epidemiological associations between early menarche and higher risks of adult disease, which are partially independent of BMI. The first studies on age at menarche identified variants intronic in LIN28B [62-64], which encodes a potent and specific regulator of let-7 microRNA processing. Experimental overexpression of its homologue Lin 28 a promotes growth, delays pubertal onset and maturation and enhances insulin sensitivity, while fetal (but not neonatal or adult) deficiency of Lin28a and Lin28b impairs results in postnatal growth defects, insulin resistance and glucose intolerance [65]. Other possible mechanisms linking puberty timing to adult disease include actions of GA$\mathrm{BA}_{\mathrm{B}}$ receptor signalling on pancreatic $\beta$-cell function [66], mitochondrial sirtuin 3 on cell responses to oxidative stress [67] and DLK1/preadipocyte factor 1 on peripheral lipid oxidation and lipid storage [68].

\section{Conclusions}

Genetic studies have underpinned our understanding of biological mechanisms that regulate puberty timing. Both monogenic studies of patients with rare disorders and polygenic studies of large population-based samples support the key role of embryonic development and signalling pathways of the hypothalamic-pituitary axis, as well as important links between energy homeostasis, growth and development, which re-enforce the critical connection between energy balance and reproduction. While further evidence is needed to identify and confirm the causal genes at GWAS loci, pathway-based analyses provide strong evidence for mechanisms such as retinoid signalling and lysine-specific demethylases.

Finally, the timing of childhood growth and development has been much debated as a trait with putative selection advantages. In adverse settings characterized by extremely high child and early adult mortality, early puberty and onset of reproductive maturity may increase fecundity despite the side effect of short adult height [69]. Conversely, in a recent analysis genetic variants associated with later age at menarche show evidence of positive selection in both European and African populations [70]. The enrichment of menarche-associated SNPs in imprinted gene regions further supports puberty timing as a selective trait and indicates parental conflict in this evolutionary perspective. Genomic imprinting results in monoallelic expression exclusively from the maternal or paternal copy. It affects only around 100 mammalian genes and supposedly evolved to control the dosage of developmentally important genes such as those that regulate placentation, embryonic growth, infant feeding, or parenting [71]. It has been hypothesized that paternal fitness is enhanced by maximizing resource allocation to his offspring, while maternal fitness is enhanced by reserving resources for her subsequent children [72, 73]. In addition to such reflective insights, the increasing evidence implicating epigenetic mechanisms in puberty timing and reproductive function may inform future understanding of early life programming and therapeutic strategies.
References

Neuroendocrinology 2015;102:247-255 DOI: $10.1159 / 000431023$
1 Parent AS, et al: The timing of normal puberty and the age limits of sexual precocity: variations around the world, secular trends, and changes after migration. Endocr Rev 2003;24: 668-693.

$\checkmark 2$ Elks CE, et al: Age at menarche and type 2 diabetes risk: The EPIC-InterAct study. Diabetes Care 2013;36:3526-3534.

3 Lakshman R, et al: Early age at menarche associated with cardiovascular disease and mortality. J Clin Endocrinol Metab 2009;94:49534960. 
4 Morris DH, Jones ME, Schoemaker MJ, Ashworth A, Swerdlow AJ: Secular trends in age at menarche in women in the UK born 190893: results from the Breakthrough Generations Study. Paediatr Perinat Epidemiol 2011; 25:394-400.

5 Hosokawa M, Imazeki S, Mizunuma $\mathrm{H}$, Kubota T, Hayashi K: Secular trends in age at menarche and time to establish regular menstrual cycling in Japanese women born between 1930 and 1985. BMC Womens Health 2012;12:19.

6 Cabanes A, et al: Decline in age at menarche among Spanish women born from 1925 to 1962. BMC Public Health 2009;9:449.

7 Euling SY, et al: Examination of US pubertytiming data from 1940 to 1994 for secular trends: panel findings. Pediatrics 2008; 121(suppl):S172-S191.

8 Adanu RMK, et al: Secular trends in menarcheal age among Ghanaian women in Accra. J Obstet Gynaecol 2006;26:550-554.

-9 Silva HP, Padez C: Secular trends in age at menarche among Caboclo populations from Pará, Amazonia, Brazil: 1930-1980. Am J Hum Biol 2006; 18:83-92.

$>_{10}$ Sørensen K, Aksglaede L, Petersen JH, Juul A: Recent changes in pubertal timing in healthy Danish boys: associations with body mass index. J Clin Endocrinol Metab 2010;95:263270.

11 Kaplowitz P: Pubertal development in girls: secular trends. Curr Opin Obstet Gynecol 2006; 18:487-491.

$\checkmark 12$ Herbison AE: Genetics of puberty. Horm Res 2007;68(suppl 5):75-79.

13 Valdes-Socin H, et al: Reproduction, smell, and neurodevelopmental disorders: genetic defects in different hypogonadotropic hypogonadal syndromes. Front Endocrinol (Lausanne) 2014;5:109.

14 Layman LC, et al: Delayed puberty and hypogonadism caused by mutations in the folliclestimulating hormone beta-subunit gene. $\mathrm{N}$ Engl J Med 1997;337:607-611.

$\checkmark 15$ Aittomäki, K, et al: Mutation in the folliclestimulating hormone receptor gene causes hereditary hypergonadotropic ovarian failure. Cell 1995;82:959-968.

16 Tüttelmann F, et al: Combined effects of the variants $F S H B-211 \mathrm{G}>\mathrm{T}$ and $F S H R 2039 \mathrm{~A}>\mathrm{G}$ on male reproductive parameters. J Clin Endocrinol Metab 2012;97:3639-3647.

17 Weiss J, et al: Hypogonadism caused by a single amino acid substitution in the beta subunit of luteinizing hormone. N Engl J Med 1992;326:179-183.

-18 Teles MG, et al: A GPR54-activating mutation in a patient with central precocious puberty. N Engl J Med 2008;358:709-715.

19 Silveira LG, et al: Mutations of the KISS1 gene 37 in disorders of puberty. J Clin Endocrinol Metab 2010;95:2276-2280.

20 Abreu AP, et al: Central precocious puberty caused by mutations in the imprinted gene MKRN3. N Engl J Med 2013;368:2467-2475.
21 Schreiner F, Gohlke B, Hamm M, Korsch E, Woelfle J: MKRN3 mutations in familial central precocious puberty. Horm Res Paediatr 2014;82:122-126.

22 Macedo DB, et al: Central precocious puberty that appears to be sporadic caused by paternally inherited mutations in the imprinted gene makorin ring finger 3. J Clin Endocrinol Metab 2014;99:E1097-E1103.

23 Bernard G, et al: Mutations of POLR3A encoding a catalytic subunit of RNA polymerase Pol III cause a recessive hypomyelinating leukodystrophy. Am J Hum Genet 2011;89:415423.

24 Margolin DH, et al: Ataxia, dementia, and hypogonadotropism caused by disordered ubiquitination. N Engl J Med 2013;368:19922003.

25 Shi Y, et al: Identification of CHIP as a novel causative gene for autosomal recessive cerebellar ataxia. PLoS One 2013;8:e81884.

26 Shi C.-H, et al: Ataxia and hypogonadism caused by the loss of ubiquitin ligase activity of the $\mathrm{U}$ box protein CHIP. Hum Mol Genet 2014;23:1013-1024.

27 Montague CT, et al: Congenital leptin deficiency is associated with severe early-onset obesity in humans. Nature 1997;387:903-908.

28 Chehab FF: Early onset of reproductive function in normal female mice treated with leptin. Science 1997;275:88-90.

29 Strobel A, Issad T, Camoin L, Ozata M, Strosberg AD: A leptin missense mutation associated with hypogonadism and morbid obesity. Nat Genet 1998;18:213-215.

-30 Clement KA: Mutation in the human leptin receptor gene causes obesity and pituitary dysfunction. Nature 1998;392:398-401.

- 31 Farooqi IS, et al: Beneficial effects of leptin on obesity, $\mathrm{T}$ cell hyporesponsiveness, and neuroendocrine/metabolic dysfunction of human congenital leptin deficiency. J Clin Invest 2002;110:1093-1103.

32 Cheung CC, et al: Leptin is a metabolic gate for the onset of puberty in the female rat. Endocrinology 1997;138:855-858.

33 Farooqi IS, et al: Clinical and molecular genetic spectrum of congenital deficiency of the leptin receptor. N Engl J Med 2007;356:237247.

34 Weedon MN, et al: An in-frame deletion at the polymerase active site of $P O L D 1$ causes a multisystem disorder with lipodystrophy. Nat Genet 2013;45:947-950.

35 Perry JRB, et al: Parent-of-origin-specific allelic associations among 106 genomic loci for age at menarche. Nature 2014;514:92-97.

36 Locke AE, et al: Genetic studies of body mass index yield new insights for obesity biology. Nature 2015;518:197-206.

37 Shungin D, et al: New genetic loci link adipose and insulin biology to body fat distribution. Nature 2015;518:187-196.

38 Wood AR, et al: Defining the role of common variation in the genomic and biological architecture of adult human height. Nat Genet 2014;46:1173-1186.
9 Cousminer DL, et al: Genome-wide association study of sexual maturation in males and females highlights a role for body mass and menarche loci in male puberty. Hum Mol Genet 2014;23:4452-4464.

40 Kaprio J, et al: Common genetic influences on BMI and age at menarche. Hum Biol 1995;67: 739-753.

41 Speliotes EK, et al: Association analyses of 249,796 individuals reveal 18 new loci associated with body mass index. Nat Genet 2010; 42:937-948.

42 Randall JC, et al: Sex-stratified genome-wide association studies including 270,000 individuals show sexual dimorphism in genetic loci for anthropometric traits. PLoS Genet 2013; 9:e1003500

43 Juul A, Magnusdottir S, Scheike T, Prytz S, Skakkebaek NE: Age at voice break in Danish boys: effects of pre-pubertal body mass index and secular trend. Int J Androl 2007;30:537542.

44 Lee JM, et al: Body mass index and timing of pubertal initiation in boys. Arch Pediatr Adolesc Med 2010;164:139-144.

45 Elks CE, et al: Thirty new loci for age at menarche identified by a meta-analysis of genome-wide association studies. Nat Genet 2010;42:1077-1085.

46 Horikoshi M, et al: New loci associated with birth weight identify genetic links between intrauterine growth and adult height and metabolism. Nat Genet 2013;45:76-82.

47 D'Aloisio AA., DeRoo LA., Baird DD, Weinberg CR, Sandler, DP: Prenatal and infant exposures and age at menarche. Epidemiology 2013;24:277-284.

48 Mueller NT, et al: Earlier menarche is associated with fatty liver and abdominal ectopic fat in midlife, independent of young adult BMI: the CARDIA study. Obesity (Silver Spring) 2015;23:468-474.

49 Willemsen RH, Elleri D, Williams RM, Ong KK, Dunger DB: Pros and cons of GnRHa treatment for early puberty in girls. Nat Rev Endocrinol 2014;10:352-363.

50 Onland-Moret NC, et al: Age at menarche in relation to adult height: the EPIC study. Am J Epidemiol 2005; 162:623-632.

51 Ong KK, et al: Timing of voice breaking in males associated with growth and weight gain across the life course. J Clin Endocrinol Metab 2012;97:2844-2852.

52 Hoggart CJ, et al: Novel approach identifies SNPs in SLC2A10 and KCNK9 with evidence for parent-of-origin effect on body mass index. PLoS Genet 2014;10:e1004508.

53 Peters J: The role of genomic imprinting in biology and disease: an expanding view. Nat Rev Genet 2014;15:517-530

54 Lomniczi A, et al: Epigenetic control of female puberty. Nat Neurosci 2013;16:281-289.

55 Kurian JR, Terasawa E: Epigenetic control of gonadotropin releasing hormone neurons. Front Endocrinol (Lausanne) 2013;4:61. 
56 Kanherkar RR, Bhatia-Dey N, Csoka AB: Epigenetics across the human lifespan. Front Cell Dev Biol 2014;2:49.

57 Jackson RS, et al: Obesity and impaired prohormone processing associated with mutations in the human prohormone convertase 1 gene. Nat Genet 1997;16:303-306.

58 Tucker RP, et al: Teneurin-2 is expressed in tissues that regulate limb and somite pattern formation and is induced in vitro and in situ by FGF8. Dev Dyn 2001;220:27-39.

59 Hoch RV, Soriano P: Context-specific requirements for Fgfr1 signaling through Frs2 and Frs3 during mouse development. Development 2006;133:663-673.

-60 Nagl F, et al: Retinoic acid-induced nNOS expression depends on a novel PI3K/Akt/DAX1 pathway in human TGW-nu-I neuroblastoma cells. Am J Physiol Cell Physiol 2009; 297:C1146-C1156.

61 Cho S, Cho H, Geum D, Kim K: Retinoic acid regulates gonadotropin-releasing hormone $(\mathrm{GnRH})$ release and gene expression in the rat hypothalamic fragments and GT1-1 neuronal cells in vitro. Brain Res Mol Brain Res 1998; 54:74-84.

62 Perry JRB, et al: Meta-analysis of genomewide association data identifies two loci influencing age at menarche. Nat Genet 2009;41: 648-650.

63 Ong KK, et al: Genetic variation in LIN28B is associated with the timing of puberty. Nat Genet 2009;41:729-733.
$64 \mathrm{He}$ C, et al: A large-scale candidate gene association study of age at menarche and age at natural menopause. Hum Genet 2010;128: 515-527.

65 Shinoda G, et al: Fetal deficiency of lin28 programs life-long aberrations in growth and glucose metabolism. Stem Cells 2013;31: 1563-1573.

66 Tian J, Dang H, Nguyen AV, Chen Z, Kaufman DL: Combined therapy with GABA and proinsulin/alum acts synergistically to restore long-term normoglycemia by modulating T-cell autoimmunity and promoting $\beta$-cell replication in newly diabetic NOD mice. Diabetes 2014;63:3128-3134.

67 Kumar S, Lombard DB: Mitochondrial sirtuins and their relationships with metabolic disease and cancer. Antioxid Redox Signal 2015; 22:1060-1077.

68 Charalambous $\mathrm{M}$, et al: DLK1/PREF1 regulates nutrient metabolism and protects from steatosis. Proc Natl Acad Sci USA 2014;111: 16088-16093.

69 Migliano AB, Vinicius L, Lahr MM: Life history trade-offs explain the evolution of human pygmies. Proc Natl Acad Sci USA 2007; 104:20216-20219.

70 Fagny M, et al: Exploring the occurrence of classic selective sweeps in humans using whole-genome sequencing data sets. Mol Biol Evol 2014;31:1850-1868.
71 Bartolomei MS, Ferguson-Smith AC: Mammalian genomic imprinting. Cold Spring Harb Perspect Biol 2011;3:a002592.

72 Wilkins JF, Haig D: What good is genomic imprinting: the function of parent-specific gene expression. Nat Rev Genet 2003;4:359368.

73 Crespi B: The evolutionary biology of child health. Proc Biol Sci 2011;278:1441-1449.

74 Lango Allen $\mathrm{H}$, et al: Hundreds of variants clustered in genomic loci and biological pathways affect human height. Nature 2010;467: 832-838.

75 Scott RA, et al: Large-scale association analyses identify new loci influencing glycemic traits and provide insight into the underlying biological pathways. Nat Genet 2012;44:9911005.

76 Manning AK, et al: A genome-wide approach accounting for body mass index identifies genetic variants influencing fasting glycemic traits and insulin resistance. Nat Genet 2012; 44:659-669.

77 Morris AP, et al: Large-scale association analysis provides insights into the genetic architecture and pathophysiology of type 2 diabetes. Nat Genet 2012;44:981-990.

78 Heid IM, et al: Meta-analysis identifies 13 new loci associated with waist-hip ratio and reveals sexual dimorphism in the genetic basis of fat distribution. Nat Genet 2010;42:949960 . 